\title{
The Glycine-Rich Motif of Pyrococcus abyssi DNA Polymerase D Is Critical for Protein Stability
}

\author{
Benoît Castrec ${ }^{1,2}$, Sébastien Laurent ${ }^{1,2}$, Ghislaine Henneke ${ }^{1,2,{ }^{*}}$, Didier Flament ${ }^{1,2}$ and Jean-Paul \\ Raffin $^{1,3}$
}

\footnotetext{
${ }^{1}$ Université de Bretagne Occidentale, UMR 6197, Laboratoire de Microbiologie et Environnements Extrêmes, BP 70, F-29280 Plouzané, France

2 IFREMER, UMR 6197, Laboratoire de Microbiologie et Environnements Extrêmes, BP 70, F-29280 Plouzané, France

${ }^{3}$ CNRS, UMR 6197, Laboratoire de Microbiologie et Environnements Extrêmes, BP 70, F-29280 Plouzané, France
}

*: Corresponding author : G. Henneke, email address : Ghislaine.Henneke@ifremer.fr

\begin{abstract}
:
A glycine-rich motif described as being involved in human polymerase $\delta$ proliferating cell nuclear antigen (PCNA) binding has also been identified in all euryarchaeal DNA polymerase D (Pol D) family members. We redefined the motif as the (G)-PYF box. In the present study, Pol D (G)-PYF box motif mutants from Pyrococcus abyssi were generated to investigate its role in functional interactions with the cognate PCNA. We demonstrated that this motif is not essential for interactions between PabPol D $(P$. abyssi Pol D) and PCNA, using surface plasmon resonance and primer extension studies. Interestingly, the (G)-PYF box is located in a hydrophobic region close to the active site. The (G)-PYF box mutants exhibited altered DNA binding properties. In addition, the thermal stability of all mutants was reduced compared to that of wild type, and this effect could be attributed to increased exposure of the hydrophobic region. These studies suggest that the (G)-PYF box motif mediates intersubunit interactions and that it may be crucial for the thermostability of PabPol D.
\end{abstract}

Keywords: DNA replication; DNA polymerase D, (G)-PYF box; Archaea; thermostability

Abbreviations: PCNA, proliferating cell nuclear antigen; Pol D, DNA polymerase D; Pol B, DNA polymerase B; PIP, PCNA-interacting protein; EMSA, electrophoresis mobility shift assay; SPR, surface plasmon resonance; TBS-T, Tris-buffered saline; EDTA, ethylenediaminetetraacetic acid 


\section{Introduction}

The processivity of DNA polymerase has been conserved among prokaryotes and eukaryotes. A double or triple replicative DNA polymerase is recruited for DNA synthesis in Escherichia coli (DNA polymerase III), ${ }^{1}$ whereas two replicases, DNA polymerases $\delta$ and $\varepsilon$, are found in eukaryotes. The picture is more confusing in Archaea because the spectra of DNA polymerases differ significantly between the Crenarchaea and Euryarchaea sub-domains. Crenarchaea possess two to three DNA polymerase B (Pol B) family members, ${ }^{2-4}$ whereas one Pol B and one DNA polymerase D (Pol D) family member are found in Euryarchaea ${ }^{5-7}$ Recently, Pol D has also been detected in the genomes of Candidatus Korarchaeum cryptofilum, ${ }^{8}$ Nanoarchaeum equitans, ${ }^{9}$ and Cenarchaeum symbiosum. ${ }^{10}$ During the last 10 years, some biochemical characterizations of Pol D have been published, ${ }^{7,11,12}$ but no structural information is yet available. Pol D is composed of two subunits, DP1 and DP2, ${ }^{5}$ and is described as a heterodimer, ${ }^{7}$ though some studies have shown that PhoPol D can form a heterotetrameric structure containing two large subunits and two small subunits. ${ }^{12}$ The small subunit, DP1, exhibits strong homology with the non-catalytic B-subunits of the eukaryotic DNA polymerases $\alpha, \delta$, and $\varepsilon .{ }^{13}$ This subunit harbors the $3^{\prime} \rightarrow 5^{\prime}$ ' exonuclease activity, and the large subunit, DP2, is the DNA polymerase subunit, ${ }^{11}$ in which Asp1122 and Asp1124 have been identified as catalytic residues. ${ }^{12}$ In addition, the $3^{\prime} \rightarrow 5^{\prime}$ exonuclease activity is regulated by the N-terminal extremity of DP $1{ }^{7,14}$ and stimulated by interaction with DP $2 .{ }^{15}$ The interactions between the two subunits might also be essential for activity. ${ }^{6}$

The role of DNA polymerases acting at the euryarchaeal replication fork, and the functional interactions of these DNA polymerases with the processivity factor, has been studied. When more than one replicative DNA polymerase is present, questions about their involvement in leading and lagging strand synthesis are raised ${ }^{16-18}$. Recent data demonstrated that Pol D is an essential DNA polymerase. ${ }^{19}$ Moreover, in a previous study, we showed that PabPol D is capable of RNA primer elongation, making it a good candidate for the initiation of DNA synthesis at the leading and lagging strands. ${ }^{20,21}$ Also, on the leading strand, we proposed a DNA polymerase switch from Pol D to Pol B, ${ }^{21}$ 
suggesting that $P a b \mathrm{Pol} \mathrm{B}$ and $P a b \mathrm{Pol} \mathrm{D}$ cooperate to perform efficient and coordinated DNA synthesis.

PCNA stimulates Pol B and Pol D on a circular DNA template in the absence of the RF-C. ${ }^{2}$ Also, PCNA interacts strongly with DP2 and weakly with DP1 in Pyrococcus furiosus, ${ }^{22}$ and the N-terminal region (1-200) of DP1 interacts with PCNA in Pyrococcus horikoshii. ${ }^{23}$ The highly conserved PCNA interacting protein (PIP) box ${ }^{24}$ is commonly described as a specific structural determinant of protein interactions with the sliding clamp. Nevertheless, the PIP box is not always sufficient to imply the requirement of additional structural elements. This statement is exemplified by our recent findings that Pol D from P. abyssi requires two PIP box motifs localized on N- and C-terminus to interact with PCNA. ${ }^{25}$ In addition, a region proximal to the core-conserved domain can significantly contribute to the physical PCNA interaction. ${ }^{26}$ Recent studies have also shown that PCNA binding of PIP box peptides is not only dependent on their structure, but also on their sequence. ${ }^{27}$ Furthermore, in eukaryotes, the binding of DNA polymerase $\delta$ to PCNA has been shown to be mediated by a glycinerich region (GX4GX8GX3YFY). ${ }^{28}$

In this study, we focused on a glycine-rich motif of PabPol D that is similar to the motif in human DNA polymerase $\delta$ and its implication on PCNA-binding. Finally, we compared the interactions between both subunits of wild-type and mutants. Our data suggest that this glycine-rich motif plays a role in the stability of $P a b P o l \mathrm{D}$. 


\section{Results}

\section{Another putative PCNA-binding motif in euryarchaeal DNA polymerases?}

In order to demonstrate that the $\mathrm{N}$ - and the $\mathrm{C}$-terminal parts are the sole structural determinants for PCNA-binding, ${ }^{25}$ we carefully inspected other putative PCNA-binding motifs in Pol D. We found that the glycine-rich motif identified ${ }^{28}$ is present in the core of the large subunits of euryarchaeal Pol D and eukaryotic DNA polymerase $\delta$ (Figure 1a). We suggest calling this motif the (G)-PYF box because these three amino acids are highly conserved.

\section{The (G)-PYF box does not favor physical and functional interactions between PabPol D and PabPCNA}

The (G)-PYF box of PabPol D was mutated in two different ways, with the resulting proteins termed PabPol Dmut1 and PabPol Dmut2 (Figure 1b). The DNA-dependent interactions of the mutant proteins with PabPCNA were then investigated; their physical interactions were similar to that of PabPol D (Figure 2), suggesting that the (G)-PYF box is not essential for PabPCNA recognition. The functional interactions of the mutant proteins with PabPCNA were also investigated (Figure 3). The addition of $P a b P C N A$ led to full-length products for the different ratios of PabPCNA and mutated or wild-type $P a b \mathrm{Pol} \mathrm{D}$ tested (Figure 3 ). The primer elongation activity of $P a b \mathrm{Pol} \mathrm{D}$ in the presence of PabPCNA was also tested in the presence of the competitor peptide PYFD, corresponding to the primary structure of the (G)-PYF box. Stimulation of full DNA synthesis by PabPol D was still observed in the presence of the peptide, confirming that the functional interaction between PabPol D and PabPCNA is (G)-PYF box-independent (data not shown). Definitively, the (G)-PYF box motif is not required for PCNA binding.

\section{Wild-type and mutant PabPol D bind differently to DNA}

In order to describe binding capacity of PabPol D, PabPol Dmut1, PabPol Dmut2 to DNA substrates, EMSA experiments were carried out in the presence of increasing amount of proteins (Figure 4). In 
the presence of PabPol D (lanes 2-4), two shifted species appeared (arrows 1 and 2) with the predominant complex related to the highest species. SPR experiments have shown afterwards that an amount of protein corresponding to the weight of two PabPolD molecules could bind one primedDNA template with an affinity constant of $56 \mathrm{nM}$ (see Supplementary material). Thus, we assumed that the higher migrating complex corresponded to the 2:1 DNA/protein complex (arrow 1) and the barely detectable shifted species was equivalent to the 1:1 DNA/protein complex (arrow 2). Such multiple binding modes can be envisioned as PabPol D has the capacity to bind both primed-DNA template and single-stranded DNA. ${ }^{20}$ For PabPol Dmut1 (lanes 8-10), one single shifted species was observed corresponding to the 1:1 band shift of $\mathrm{PabPol}$ D (arrow 2). Affinity constant of that complex was too low $(>1 \mu \mathrm{M})$ to allow a fine determination by SPR approach. For PabPol Dmut2 (lanes 5-7), two distinct shifted species were obtained; one with a similar shift to the 1:1 DNA/PabPol D complex and the second with a lower band shift (arrow 3), indicating that a protein of lower molecular weight than the heterodimeric PabPol D could still retain DNA binding. Finally, SPR calculations gave a global $\mathrm{K}_{\mathrm{D}}$ of $404 \mathrm{nM}$ for the PabPol Dmut2. Overall, data obtained by SPR measurements significantly correlated to the observed shifted species formed over the range of the protein concentration tested by EMSA. These results clearly show that mutation of $(\mathrm{G})$-PYF box modulates DNA binding properties, mainly by decreasing DNA binding ability.

\section{Physical and functional interaction between DP1 and DP2}

His-tagged wild-type DP1 was produced and purified as described in "Experimental procedures". DP2 of PabPol D, PabPol Dmut1, and PabPol Dmut2 were expressed separately in the E. coli HMS 174 strain $(50 \mathrm{ml})$. The interactions between both subunits were investigated by pull-down assays using DP1 as bait and immunoblotting for DP2 (Figure 5a). Physical interactions of the large DP2 and small DP1 subunits were similarly preserved in PabPol D and PabPol Dmut1. However, the interaction between DP1 and DP2 of PabPol Dmut2 was weaker, suggesting that the (G)-PYF box may participate in inter-subunit interactions, even if it is not essential.

Next, wild-type DP1 and DP2 of PabPol D, PabPol Dmut1, and PabPol Dmut2, expressed separately in the E. coli HMS 174 strain, were mixed together and tested using primer extension assays (Figure 
5b). DP1 and DP2 did not express primer extension activity alone (Figure 5b, lanes 2-5). The primer extension activity of DP1 mixed with DP2 of PabPol Dmut1 was similar to that of wild-type DP2, but it was lower with DP2 from PabPol Dmut2 (Figure 5b, lanes 6-8), suggesting that the PabPol D complex is less stable with mutations in the (G)-PYF box. In addition, it was not possible to reconstitute the $3^{\prime} \rightarrow 5^{\prime}$ exonuclease activity under any conditions (data not shown).

\section{The (G)-PYF box motif of PabPol D is involved in the thermal stability of the enzyme}

\section{Nile Red fluorescence}

Nile Red fluorescence is sensitive to the polarity of its environment and can be used as a probe for the presence of hydrophobic regions in proteins. ${ }^{29}$ As a consequence, Nile Red is regularly employed to probe changes in protein conformation and the exposure of hydrophobic surfaces. ${ }^{30}$ The spectra of thermally treated PabPol D showed a very weak blue shift from 660 to 658 , as well as a weak increase in fluorescence intensity (Figure 6a). Thus, wild-type Pol D appears to be thermally stable. On the contrary, the spectra of thermally treated samples of PabPol Dmut1 showed a high blue shift from 660 to 646 and a high increase in fluorescence intensity (Figure 6b). Similarly, a blue shift from 656 to 643 was observed for PabPol Dmut2, which was progressive with temperature treatment (Figure 6c). These results suggest that higher heating results in more hydrophobic regions of PabPol Dmut1 and PabPol Dmut2 being exposed, contrary to what was observed for wild-type PabPol D. Thus, we hypothesized that the (G)-PYF box mutants conferred a lower thermal stability to PabPol D.

\section{Primer extension assays}

The primer extension activity of thermally treated PabPol D and (G)-PYF box mutants are shown in Figure 6d. Wild-type PabPol D was less active after heating to $100^{\circ} \mathrm{C}$ (Figure 6d, lanes 2-5), whereas PabPol Dmut1 and PabPol Dmut2 progressively lost their DNA polymerase activity from $80^{\circ} \mathrm{C}$ (Figure 6d, lanes 6-13). More precisely, after heating to $90^{\circ} \mathrm{C}$, full-length products were less prevalent with PabPol Dmut1 and PabPol Dmut2 (Figure 6d, lanes 8 and 12). After heating to $100^{\circ} \mathrm{C}, P a b P o l$ Dmut1 elongated small products but PabPol Dmut2 was inactive (Figure 6d, lanes 9 and 13). Thus, heating induced a decrease in the polymerase activity of the mutants compared to wild-type DNA 
polymerase D. This finding confirms that the (G)-PYF box motif of PabPol D is involved in the stability of the enzyme. 


\section{Discussion}

We investigated a motif that was previously shown to be involved in the direct interaction between PCNA and the p125 catalytic subunit of mammalian DNA polymerase $\delta .^{28,31}$ This motif is found in all euryarchaeal DNA polymerase D (Figure 1a) and in some p125 catalytic subunits of eukaryotic DNA polymerase $\delta$. We proposed calling the motif the (G)-PYF box because of the occurrence of a conserved amino-acid triplet (Pro, Tyr, Phe).

In our study, PabPol D (G)-PYF box mutants retain stimulation by PabPCNA, suggesting that, at least in Archaea, the (G)-PYF box motif is not involved in the interaction with PCNA. We also characterized the role of this glycine-rich motif. Firstly, we showed that the two (G)-PYF box mutants, PabPol Dmut1 and PabPol Dmut2, interact differently with DNA compared to wild-type PabPol D. This behavior can be explained by two points of view. On the one hand, most crenarchaeal Pol B3, euryarchaeal Pol B, and crenarchaeal Pol B1 possess a motif at their N-terminus that is partially related to the (G)-PYF box (data not shown). Interestingly, many studies have reported a sequence including this amino-acid triplet in the N-terminal domains of archaeal DNA polymerases that is clearly positioned at a region interacting with DNA. ${ }^{32}$ Indeed, the proline and tyrosine are highly conserved in the uracil binding pocket responsible for uracil recognition by the archaeal Pol B. ${ }^{33,34}$ However, the other residues defining the uracil pocket are not found in PabPol D. Also, we do not yet know if uracil detection activity is present in PabPol D as it is in Pol B. On the other hand, the (G)-PYF box motif is located in the vicinity of the two catalytic residues, D956 and D958. ${ }^{7}$ No influence of mutations in the (G)-PYF box motif on polymerase activity was observed. Thus, we can easily imagine that mutations in the (G)-PYF box motif involve a conformational change at the level of this sequence that does not affect the active site of Pol D.

Results obtained from EMSA clearly show that DNA binding capacity is largely affected by those mutations. PabPol Dmut1 demonstrates an important decrease of its binding affinity toward primedDNA template. Moreover, a similar behavior was observed with PabPol Dmut2 excepted that an additional lower band shift appeared. Here, we can hypothesize that only one subunit of PabPol Dmut2 retains DNA binding ability. Consequently, further experiments are required to identify the 
remaining bound subunit and its underlying binding mechanism. All together, these analyses pointed out a lower DP1/DP2 stability within the heterodimeric PabPol Dmut2 mutant.

Interestingly, in P. horikoshii, the domain (792-1163) of the large subunit of Pol D contains three hydrophobic regions. ${ }^{23}$ As the primary structures of the DNA polymerases of $P$. horikoshii and $P$. abyssi are analogous, particularly at the level of the (G)-PYF box, the (G)-PYF box motif (927-947) belongs to one of the hydrophobic regions.

In order to determine the implication of the (G)-PYF box motif in inter-subunit stability, the small and large subunits of the wild-type and mutant PabPol D were expressed separately. The physical and functional interactions between DP1 and DP2 of PabPol Dmut2 seem to be weaker than those of PabPol Dmut1. Therefore, increasing the number of mutations in this zone leads to a progressive loss of complex stability. Finally, if the (G)-PYF box is not an essential motif participating in inter-subunit assembly within the range of amino acids 792-1163 of the large subunit of PhoPol D, mutations in the (G)-PYF box motif, particularly at the level of tyrosine 946 and phenylalanine 947, induce radical changes in the conformation of the protein. Heating experiments confirmed this assumption. Proteins with mutated (G)-PYF box motifs were rapidly degraded, demonstrating their weak thermostability compared to the wild-type protein. In addition, heating induced decreased the DNA polymerase activity of the mutant enzymes. Hydrophobic forces are major contributors to molecular folding and thermostability. ${ }^{35,36}$ This result corroborates the studies on P. horikoshii that found that the domain (792-1163) plays a role in the stability of the PhoPol D complex and in polymerase activity. ${ }^{23} \mathrm{We}$ show that, inside this domain, the (G)-PYF box has a major role in Pol D stability. 


\section{Materials and Methods}

\section{Recombinant proteins}

PabPol D and PabPCNA were prepared as previously described. ${ }^{7,37}$ In PabPol Dmut1 and PabPol Dmut2, the glycine at positions 934 and 941 (PabPol Dmut1) and tyrosine 946 and phenylalanine 947 (PabPol Dmut2) were substituted by alanine. The two mutants were obtained using the following oligonucleotides (mutated codons are underlined): 5'-

AGGATAATAGCCTTTGTCGATGCCCTAGTTGCTTACG CT-3’ and 3’-

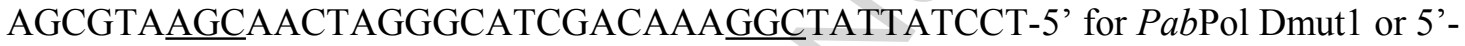
GCCCTAGTTGGTTACGCTCACCCCGCCGCCCACGCTGC G-3' and 3'CGCAGCGTGGGCGGCGGGgtgAGCGTAACCAACTAGGGC-5' for PabPol Dmut2. The PCR products were cloned into the pET26b+ plasmid (Promega), and the entire nucleotide sequence was confirmed by sequencing. The two mutants were purified as previously described for PabPol D. ${ }^{7}$ DP1 and DP2 of PabPol D, DP2 of PabPol Dmut1, and DP2 of PabPol Dmut2 constructs containing the bacteriophage $\mathrm{T} 7$ gene promoter and leader sequence were introduced together or separately into E. coli strains HMS174(DE3). The cells were grown at $37^{\circ} \mathrm{C}$ in $\mathrm{LB}$ medium with ampicillin (100 $\mathrm{mg}$ $\left.\mathrm{ml}^{-1}\right)$ or kanamycin $\left(30 \mathrm{mg} \mathrm{ml}^{-1}\right)$ with gentle shaking $(150 \mathrm{rpm})$. The cells were grown to an $\mathrm{A}_{600 \mathrm{~mm}}$ of 0.7 , and then IPTG was added to a final concentration of $1 \mathrm{mM}$ to induce the overproduction of the recombinant proteins. The cells were cultured for an additional 4 hours and centrifuged at 13,000 x $\mathrm{g}$ for $15 \mathrm{~min}$ at $4^{\circ} \mathrm{C}$. The pellets were resuspended in lysis buffer $(50 \mathrm{mM}$ Tris $\mathrm{HCl} \mathrm{pH} 8,2 \mathrm{mM} \beta$ mercaptoethanol, and protease inhibitor cocktail tablets; Complete, Roche diagnostics), and bacterial lysates were prepared by sonication ( $3 \times 15 \mathrm{~s}$ pulses). Cellular debris was removed by centrifugation at $13,000 \mathrm{x}$ g for $15 \mathrm{~min}$ at $4^{\circ} \mathrm{C}$. The supernatants were heated at $75^{\circ} \mathrm{C}$ for $10 \mathrm{~min}$, followed by centrifugation at $13,000 \mathrm{xg}$ for $15 \mathrm{~min}$ at $4^{\circ} \mathrm{C}$. The supernatants were loaded onto an SDS-PAGE Criterion XT 12\% polyacrylamide precast gel (Bio-Rad) and analyzed by Western blotting. His-tagged DP1 of PabPol D was purified as follows. In short, the extract was passed through an affinity column (His-trap, $5 \mathrm{ml} ; 1 \mathrm{ml} \mathrm{min}{ }^{-1}$ ) pre-equilibrated with $50 \mathrm{mM} \mathrm{Na}(\mathrm{P})(\mathrm{pH} 6.5)$ containing 1 
$\mathrm{mM}$ DTT and $20 \mathrm{mM}$ imidazole. The proteins were eluted by a linear gradient of imidazole up to 500 $\mathrm{mM}$. The fractions containing DP1 (detected by SDS-PAGE) were pooled and loaded onto a Hiprep 16/10 Q FF column $\left(1 \mathrm{ml} \mathrm{min}{ }^{-1}\right)$ pre-equilibrated with $50 \mathrm{mM} \mathrm{Na}(\mathrm{P})(\mathrm{pH}$ 6.5) containing $1 \mathrm{mM}$ DTT. DP1 was eluted by a linear gradient of $\mathrm{NaCl}$ up to $1 \mathrm{M}$. The fractions containing DP1 were dialyzed against $20 \mathrm{mM}$ Tris $\mathrm{HCl}(\mathrm{pH} 8)$ containing $1 \mathrm{mM}$ DTT and 20\% (v/v) glycerol, concentrated, and stored at $-20^{\circ} \mathrm{C}$.

\section{Western blotting}

Proteins separated on the SDS-PAGE Criterion XT 12\% polyacrylamide precast gel (Bio-Rad) were transferred to immune-blot polyvinylidene difluoride membranes (Bio-Rad). The blots were saturated with blocking buffer containing 5\% (w/v) skim milk in TBS-T (Tris-buffered saline: $10 \mathrm{mM}$ Tris-HCl $\mathrm{pH} 7.5,150 \mathrm{mM} \mathrm{NaCl}, 0.05 \%(\mathrm{v} / \mathrm{v})$ Tween 20$)$ for $1 \mathrm{~h}$ at room temperature. The blots were incubated for $1 \mathrm{~h}$ at room temperature with a 1:5,000 dilution of the rabbit polyclonal anti-DP1 for PabPol D and the rabbit polyclonal anti-DP2 for PabPol D in TBS-T. The membranes were then washed three times with TBS-T and incubated for $1 \mathrm{~h}$ at room temperature in a 1:10,000 dilution of horseradish peroxidase-linked anti-mouse IgG in TBS-T. The membranes were washed three times with TBS-T and the secondary antibody was detected by the enhanced chemiluminescence system ECL+ (Amersham Biosciences). The bands were visualized on a Typhoon 9400 imager (Amersham Biosciences).

\section{Primer extension assay}

The 32-nt oligonucleotide used to prepare the primed DNA substrate for the primer extension assays (5'-TGCCAAGCTTGCATGCCTGCAGGTCGACTCTA-3') was synthesized, labeled at the 5' terminus with 6-carboxyfluorescein, and purified by Eurogentec. The 32-nt oligonucleotide was either annealed to M13ssc at a 3:1 molar ratio or to an 87-nt oligonucleotide (5'-

\section{CAGGAAACAGCTATGACCATGATTACGAATTCGAGCTCGG}

TACCGGGGTCCTCTAGAGTCGACCTGCAGGCATGCAAGCTTGGCA-3') at an equimolar ratio in $10 \mathrm{mM}$ Tris- $\mathrm{HCl}\left(\mathrm{pH} 8.0\right.$ ) containing $50 \mathrm{mM} \mathrm{NaCl}$ by heating to $75^{\circ} \mathrm{C}$ for $10 \mathrm{~min}$, followed by 
cooling to room temperature. Primer extensions assays were performed in a final volume of $11 \mu 1$ containing the following components: 0.09 pmol of M13 ssc, 2 pmol of each dNTP, 0.02-1.5 pmol of PabPCNA and PabPols to be tested in the buffer (50 mM Tris-HCl pH 8.8, $1 \mathrm{mM} \mathrm{DTT,} 10 \mathrm{mM} \mathrm{KCl,}$ $2 \mathrm{mM} \mathrm{MgCl} 2$ ). Reactions were incubated at $60^{\circ} \mathrm{C}$ for $30 \mathrm{~min}$ and then stopped on ice by adding $10 \mu 1$ of stop buffer $(98 \%(\mathrm{v} / \mathrm{v})$ formamide, $0.2 \mathrm{mM}$ EDTA, $\mathrm{pH} 8.0)$. The products were heated to $95^{\circ} \mathrm{C}$ for $5 \mathrm{~min}$ and resolved for $16 \mathrm{~h}$ at $30 \mathrm{~V}$ at $4{ }^{\circ} \mathrm{C}$ on a $1 \%(\mathrm{w} / \mathrm{v})$ alkaline agarose gel $(50 \mathrm{mM} \mathrm{NaOH}, 1 \mathrm{mM}$ EDTA). Primer extension with $87 \mathrm{mer} / 32 \mathrm{mer}-\mathrm{FAM}(0.03 \mathrm{pmol})$ was performed under the same conditions and contained $6 \mathrm{pmol}$ of DP1 and DP2. The products were resolved for $5 \mathrm{~h}$ at room temperature on a $15 \%$ polyacrylamide, $7 \mathrm{M}$ urea gel. Finally, the products were visualized on a Typhoon 9400 imager (Amersham Biosciences).

\section{Surface plasmon resonance (SPR) experiments}

SPR analyses were performed with an angular shift reading mode apparatus (BIAcore X, Uppsala, Sweden and Reichert SR7000, Depew, NY) using an equivalent primer DNA immobilization approaches. Briefly, a controlled amount of 5 ' biotinylated ssDNA was allowed to bind onto a precoated streptavidin surface (SA chip, BIAcore and PEG/PEG-COOH, Reichert) and then reacted with its complementary DNA template. For each measurement, flow cell temperature was set at $25^{\circ} \mathrm{C}$.

Interactions between PabPol D, PabPol Dmut1, PabPol Dmut2 and the primer DNA/PCNA complex were realized following a decaying surface analysis format. ${ }^{20} \mathrm{~A}$ chip bearing high density of primed DNA (200 RU) was employed: PabPCNA (200 nM) was injected over the DNA chip and PabPol (200 nM) was supplemented $30 \mathrm{~s}$ after the end of the injection. Running buffer consisted of HBS-P buffer $(10 \mathrm{mM}$ Hepes $\mathrm{pH} 7.4,150 \mathrm{mM} \mathrm{NaCl}, 0.005 \%(\mathrm{v} / \mathrm{v})$ surfactant $\mathrm{P} 20)$ at a flow rate of 30 $\mu \mathrm{L} / \mathrm{min}$. All constructs were removed from surface by $60 \mathrm{~s}$ of $50 \mathrm{mM} \mathrm{NaOH}$ injection.

Interactions characterized between PabPol D, PabPol Dmut1, PabPol Dmut2 and the primed-DNA template used in EMSA experiments were realized following a single injection format. Firstly, SPR experiments were carried out on a medium density DNA chip (42 RU) to allow for high capture 
levels: concentration ranges of PabPol D, PabPol Dmut1 and PabPol Dmut2 were successively injected over the DNA chip during $15 \mathrm{~min}$, let dissociate during $15 \mathrm{~min}$ and removed from the surface by $60 \mathrm{~s}$ of $0.5 \%$ SDS injection. Running buffer consisted of PBS-T (10 mM Phosphate $\mathrm{pH} 7.4,138$ $\mathrm{mM} \mathrm{NaCl}, 2.7 \mathrm{mM} \mathrm{KCl}, 0.05 \%$ Tween 20 ) at a flow rate of $20 \mu \mathrm{L} / \mathrm{min}$. Fits of binding curves were obtained with Scrubber software (Salt Lake, UT) according to a 1:1 binding Langmuir model (see Supplementary material). Secondly, in order to limit steric hindrance on the sensor surface, a low density DNA chip (6 RU) was employed following the same process as described above. Fits of binding curves were processed according to a 2:1 binding Langmuir model considering two equivalent binding sites onto the DNA sequence (see Supplementary material).

\section{Nile Red fluorescence}

All analyses were performed using an Edinburgh FLS920 spectrofluorimeter. The cell, with a path length of $3 \mathrm{~mm}$ and a working volume of $120 \mu \mathrm{l}$, was thermostated at $20^{\circ} \mathrm{C} \pm 0.5^{\circ} \mathrm{C}$. The excitation wavelength was set at $550 \mathrm{~nm}$, and the excitation and emission slits were set at $5 \mathrm{~nm}$ and $10 \mathrm{~nm}$, respectively. Nile Red was dissolved in $\mathrm{Me}_{2} \mathrm{SO}$ to a concentration of $0.25 \mathrm{mM}$, which was added to a final concentration of $1 \mu \mathrm{M}$ in the cell containing 48 pmol samples of PabPol D, PabPol Dmut1, or PabPol Dmut2. Spectra were recorded after 5 min.

\section{Thermostability of wild-type and mutant PabPol D}

The thermal stabilities of 5 pmol PabPol D, PabPol Dmut1, or PabPol Dmut2 were monitored in dilution buffer (Tris $\mathrm{HCl} 20 \mathrm{mM} \mathrm{pH} \mathrm{7.5,} \mathrm{glycerol} \mathrm{20 \% )} \mathrm{in} \mathrm{a} \mathrm{final} \mathrm{volume} \mathrm{of} 10 \mu 1$. Each sample was incubated at $80^{\circ} \mathrm{C}, 90^{\circ} \mathrm{C}$, or $100^{\circ} \mathrm{C}$ for $5 \mathrm{~min}$ and then cooled on ice. Enzymes were assayed at 3 pmol for primer extension and at 48 pmol for Nile Red fluorescence.

\section{Pull-down assays}

His-tagged DP1 $(10 \mu \mathrm{g})$ was incubated with $4 \mu \mathrm{l}$ of magnetic beads (Dynabeads Talon, Dynal Biotech) in $100 \mu \mathrm{l}$ of binding and washing buffer (BW buffer: $50 \mathrm{mM} \mathrm{Na}(\mathrm{P}) \mathrm{pH} 8,300 \mathrm{mM} \mathrm{NaCl}$, $0.01 \%$ Tween 20) for $30 \mathrm{~min}$ at room temperature. Next, the beads were washed in BW buffer. The 
samples were incubated with DP2 for $1 \mathrm{~h}$ at room temperature and washed five times with the same buffer. Proteins were eluded from the beads in loading buffer before being loaded onto a SDS-PAGE Criterion XT 12\% polyacrylamide precast gel (Bio-Rad), and DP2 was analyzed by Western blotting.

\section{Electrophoresis mobility-shift assay (EMSA)}

Binding reactions were performed in a final volume of $20 \mu$ l containing $50 \mathrm{mM}$ Tris- $\mathrm{HCl}(\mathrm{pH}$ 8.0) containing $20 \mathrm{mM} \mathrm{NaCl}, 5 \mathrm{mM}$ EDTA, $50 \mu \mathrm{g} \mathrm{ml}^{-1} \mathrm{BSA}, 4 \%$ Ficoll, $2.5 \mathrm{pmol}$ of labeled template/primer (87mer/32mer), and 5, 10, or 15 pmol of wild-type PabPol D, PabPol Dmut1, or PabPol Dmut2. The reactions were incubated at $60^{\circ} \mathrm{C}$ for $10 \mathrm{~min}$ and cooled on ice. The samples were loaded onto a 1\% (w/v) agarose gel containing $4.5 \mathrm{mM}$ Tris $\mathrm{HCl}, 4.5 \mathrm{mM}$ Boric acid, and $2 \mathrm{mM}$ EDTA (pH 8) and run at $90 \mathrm{~V}$ for $3 \mathrm{~h}$. The products were visualized on a Typhoon 9400 imager.

\section{Sequence accession numbers}

The Entrez protein database accession numbers for the sequences used in this paper are: NP_070550 (Archaeglobus fulgidus), NP_248640 (Methanocaldococcus jannaschii), YP_184316 (Thermococcus kodakarensis), NP_125813 (Pyrococcus abyssi), NP_577748 (Pyrococcus furiosus), NP_142130 (Pyrococcus horikoshii), NP_002682 (Homo sapiens), NP_596124 (Schizosaccharomyces pombe), NP_067694 (Rattus norvegicus). 


\section{Acknowledgements}

BC and SL were supported by a grant from Brittany regional council (211-B2-9/ARED), (4852REPAR/CREATE), respectively. DF was supported by grants from the National Research Agency (ANR-07-BLAN-0371). We are grateful to Pascal Trouvé for giving us access to the BIAcore apparatus (INSERM U.613, Génétique moléculaire et génétique épidémiologique, Brest, France). 


\section{REFERENCES}

1. McInerney, P., Johnson, A., Katz, F. \& O'Donnell, M. (2007). Characterization of a triple DNA polymerase replisome. Mol. Cell 27, 527-538.

2. Cann, I.K.O. \& Ishino, Y. (1999). Archaeal DNA replication: Identifying the pieces to solve a puzzle. Genetics 152, 1249-1267.

3. Cann, I.K.O., Ishino, S. , Nomura, N., Sako, Y. \& Ishino, Y. (1999). Two family B DNA polymerases from Aeropyrum pernix, an aerobic hyperthermophilic crenarchaeote. $J$. Bacteriol. 181, 5984-5992.

4. Iwai, T., Kurosawa, N., Itoh, Y.H., Kimura, N. \& Horiuchi, T. (2000). Sequence analysis of three family B DNA polymerases from the thermoacidophilic crenarchaeon Sulfurisphaera ohwakuensis. DNA Res. 7, 243-251.

5. Cann, I.K.O., Komori, K., Toh, H., Kanai, S. \& Ishino, Y. (1998). A heterodimeric DNA polymerase: Evidence that members of Euryarchaeota possess a distinct DNA polymerase. Proc. Natl. Acad. Sci. USA 95, 14250-14255.

6. Ishino, Y., Komori, K., Cann, I.K.O., \& Koga Y., (1998). A novel DNA polymerase family found in Archaea. J. Bacteriol. 180, 2232-2236.

7. Gueguen, Y., Rolland, J.L., Lecompte, O., Azam, P., Le Romancer, G., Flament, D., Raffin, J.P. et al. (2001). Characterization of two DNA polymerases from the hyperthermophilic euryarchaeon Pyrococcus abyssi. Eur. J. Biochem. 268, 5961-5969.

8. Elkins, J.G., Podar, M., Graham, D.E., Makarova, K.S., Wolf, Y., Randau, L., Hedlund, B.P. et al. (2008). A korarchaeal genome reveals insights into the evolution of the Archaea. Proc. Natl. Acad. Sci. USA 105, 8102-8107. 
9. Waters, E., Hohn, M.J., Ahel, I., Graham, D.E., Adams, M.D., Barnstead, M., Beeson, K.Y. et al. (2003). The genome of Nanoarchaeum equitans: Insights into early archaeal evolution and derived parasitism. Proc. Natl. Acad. Sci. USA 100, 12984-12988.

10. Hallam, S.J., Konstantinidis, K.T., Putnam, N., Schleper, C., Watanabe, Y., Sugahara, J., Preston, C. et al. (2006). Genomic analysis of the uncultivated marine crenarchaeote Cenarchaeum symbiosum. Proc. Natl. Acad. Sci. USA 103, 18296-18301.

11. Uemori, T., Sato, Y., Kato, I., Doi, H., \& Ishino, Y. (1997). A novel DNA polymerase in the hyperthermophilic archaeon, Pyrococcus furiosus: gene cloning, expression and characterization. Genes Cell. 2, 499-512.

12. Shen, Y., Musti, K., Hiramoto, M., Kikuchi, H., Kawarabayashi, Y. \& Matsui, I. (2001). Invariant Asp1122 and Asp1124 are essential residues for polymerization catalysis of family D DNA polymerase from Pyrococcus horikoshii. J. Biol. Chem. 276, 27376-27383.

13. Aravind, L., Leipe, D.D. \& Koonin, E.V. (1998). Toprim - a conserved catalytic domain in type IA and II topoisomerases, DnaG-type primases, OLD family nucleases and RecR proteins. Nucl. Acids Res. 26, 4205-4213.

14. Shen, Y., Tang, X.F. \& Matsui, I. (2003). Subunit interaction and regulation of activity through terminal domains of the family D DNA polymerase from Pyrococcus horikoshii. J. Biol. Chem. 278, 21247-21257.

15. Shen, Y.L., Tang, X.F., Yokoyama, H., Matsui, E. \& Matsui, I. (2004). A 21-amino acid peptide from the cysteine cluster II of the family D DNA polymerase from Pyrococcus horikoshii stimulates its nuclease activity which is Mre11-like and prefers manganese ion as the cofactor. Nucl. Acids Res. 32, 158-168.

16. Waga, S \& Stillman, B. (1998). The DNA replication fork in eukaryotic cells. Annu. Rev. Biochem. 67, 721-751. 
17. Hübscher, U., Maga, G. \& Spadari, S. (2002). Eukaryotic DNA polymerases. Annu. Rev. Biochem. 71, 133-163.

18. Fukui, T., Yamauchi, K., Muroya, T., Akiyama, M., Maki, H., Sugino, A. \& Waga, S. (2004). Distinct roles of DNA polymerases $\delta$ and $\varepsilon$ at the replication fork in Xenopus egg extracts. Genes Cell. 9, 179-191.

19. Berquist, B.R., DasSarma, P. \& DasSarma, S. (2007). Essential and non-essential DNA replication genes in the model halophilic Archaeon, Halobacterium sp NRC-I. BMC Genet. 8, 31.

20. Henneke, G., Flament, D., Hübscher, U., Querellou, J. \& Raffin, J.P. (2005). The hyperthermophilic Euryarchaeota Pyrococcus abyssi likely requires the two DNA polymerases D and B for DNA replication. J. Mol. Biol. 350, 53-64.

21. Rouillon, C., Henneke, G., Flament, D., Querellou, J. \& Raffin, J.P. (2007). DNA polymerase switching on homotrimeric PCNA at the replication fork of the Euryarchaea Pyrococcus abyssi. J. Mol. Biol. 369, 343-355.

22. Cann, I.K.O., Ishino, S., Hayashi, I., Komori, K., Toh, H., Morikawa, K. \& Ishino, Y. (1999). Functional interactions of a homolog of proliferating cell nuclear antigen with DNA polymerases in Archaea. J. Bacteriol. 181, 6591-6599.

23. Tang, X.F., Shen, Y., Matsui, E. \& Matsui, I. (2004). Domain topology of the DNA polymerase D complex from a hyperthermophilic archaeon Pyrococcus horikoshii. Biochemistry 43, 11818-11827.

24. Warbrick, E. (1998). PCNA binding through a conserved motif. Bioessays 20, 195-199. 25. Castrec, B., Rouillon, C., Henneke, G., Flament, D., Querellou, J. \& Raffin, J.P. (2009). Binding to PCNA in Euryarchaeal DNA Replication requires two PIP motifs for DNA polymerase D and one PIP motif for DNA polymerase B. J. Mol. Biol. 394, 209-218. 
26. Pohler, J.R., Otterlei, M. \& Warbrick, E. (2005). An in vivo analysis of the localisation and interactions of human p66 DNA polymerase $\delta$ subunit. BMC Mol. Biol. 6, 17.

27. Meslet-Cladiere, L., Norais, C., Kuhn, J., Briffotaux, J., Sloostra, J.W., Ferrari, E., Hübscher, U. et al. (2007). A novel proteomic approach identifies new interaction partners for proliferating cell nuclear antigen. J. Mol. Biol. 372, 1137-1148.

28. Zhang, S.J., Zeng, X.R., Zhang, P., Toomey, N.L., Chuang, R.Y., Chang, L.S. \& Lee, M.Y.W.T. (1995). A conserved region in the amino terminus of DNA polymerase $\delta$ is involved in proliferating cell nuclear antigen binding. J. Biol. Chem. 270, 7988-7992. 29. Sackett, D.L. \& Wolff, J. (1987). Nile red as a polarity-sensitive fluorescent-probe of hydrophobic protein surfaces. Anal. Biochem. 167, 228-234.

30. Febbraio, F., Andolfo, A., Tanfani, F., Briante, R., Gentile, F., Formisano, S., Vaccaro, C. et al. (2004). Thermal stability and aggregation of Sulfolobus solfataricus beta-glycosidase are dependent upon the n-epsilon-methylation of specific lysyl residues - Critical role of in vivo post-translational modifications. J. Biol. Chem. 279, 10185-10194.

31. Zhang, P., Mo, J.Y., Perez, A., Leon, A., Liu, L., Mazloum, N., Xu, H. et al. (1999). Direct interaction of proliferating cell nuclear antigen with the p125 catalytic subunit of mammalian DNA polymerase $\delta$. J. Biol. Chem. 274, 26647-26653.

32. Fogg, M.J., Pearl, L.H. \& Connolly, B.A. (2002). Structural basis for uracil recognition by archaeal family B DNA polymerases. Nature Struct. Biol. 9, 922-927.

33. Savino, C., Federici, L., Johnson, K.A., Vallone, B., Nastopoulos, V., Rossi, M., Pisani, F.M. et al. (2004). Insights into DNA replication: The crystal structure of DNA polymerase B1 from the archaeon Sulfolobus solfataricus. Structure 12, 2001-2008.

34. Franklin, M.C., Wang, J. \& Steitz, T.A. (2001). Structure of the replicating complex of a Pol $\alpha$ family DNA polymerase. Cell 105, 657-667. 
35. Goodenough, P.W. \& Jenkins, J.A. (1991). Protein engineering to change thermalstability for food enzymes. Biochem. Soc. Trans. 19, 655-662.

36. Vieille, C. \& Zeikus, G.J. (2001). Hyperthermophilic enzymes: Sources, uses, and molecular mechanisms for thermostability. Microbiol. Mol. Biol. Rev. 65, 1-43.

37. Henneke, G., Gueguen, Y., Flament, D., Azam, P., Querellou, J., Dietrich, J., Hübscher, U. et al. (2002). Replication Factor C from the hyperthermophilic archaeon Pyrococcus abyssi does not need ATP hydrolysis for clamp-loading and contains a functionally conserved RFC PCNA-binding domain. J. Mol. Biol. 323, 795-810. 


\section{FIGURE LEGENDS}

Figure 1. PYF-box, a conserved PCNA-binding motif in diverse archaeal and eukaryotic DNA polymerases.

(a) Amino acid alignment of the PYF-box. For eukaryotes, the protein-protein interaction motif of the $\mathrm{N} 2$ region of the $\mathrm{p} 125$ subunit of $\mathrm{Pol} \delta$ is shown.

(b) Mutations performed in the (G)-PYF box motif of PabPol D. The amino acid residues of the (G)PYF box are indicated by arrows. The amino acid residues that are substituted by alanine in PabPol Dmut1 and PabPol Dmut2 are underlined.

Figure 2. SPR measurements of PabPol D, PabPol Dmut1, or PabPol Dmut2 interactions with PabPCNA. At time 0, PabPCNA was injected over the DNA surface, as indicated by the grey arrow, and PabPol D, PabPol Dmut1, or PabPol Dmut2, as indicated by the black arrow. The background resulting from the injection buffer was subtracted from the data before plotting.

Figure 3 The (G)-PYF box of PabPol D is not required for functional interactions with PabPCNA. Primer extension studies were performed using M13mp18 template (90 fmol) hybridized to a 5'fluorescein-labeled primer and containing 0.3 pmol of PabPol D, 0.6 pmol of PabPol Dmut1, or 0.3 pmol of PabPol Dmut2. PabPCNA was added at three different ratios - 1:1, 1:2, and 1:3. Reactions were incubated at $60^{\circ} \mathrm{C}$ for $30 \mathrm{~min}$. Extended products were resolved on denaturing alkaline $1 \%$ agarose gels and visualized on a Typhoon 9400 imager.

Figure 4. Gel shifts demonstrate the presence of different DNA primed template/polymerase complexes for PabPol D, PabPol Dmut2 and PabPol D mut1, respectively. Incubation temperature was set at $60^{\circ} \mathrm{C}$. The increasing amounts of enzyme $(5,10$, and $15 \mathrm{pmol})$ are symbolized by a triangle. The positions of free DNA and complexes are indicated on the left. Corresponding equilibrium binding constants $\left(\mathrm{K}_{\mathrm{D}}\right)$ calculated by SPR experiments at $25^{\circ} \mathrm{C}$ are reported below. 
Figure 5. Physical and functional interactions between DP1 and DP2 of PabPol D, PabPol Dmut1, and PabPol Dmut2.

(a) Physical interactions tested by pull-down assays. His-tagged DP1 (10 pmol) was fixed to magnetic beads, and 10 pmol of DP2 from PabPol D, PabPol Dmut1, and PabPol Dmut2 was added in $200 \mu 1$ of binding and washing buffer. Proteins were eluted, loaded on SDS-PAGE, and the presence of DP2 determined by Western blot. C, Western blot control.

(b) Primer extension assays of DP1 reconstituted in vitro with DP2 of PabPol D, PabPol Dmut1, or PabPol Dmut2. Primer extension studies were performed with the 87-nt (30 fmol) template hybridized to a 5'-fluorescein-labeled primer and containing 6 pmol each of DP1 and DP2 from PabPol D, PabPol Dmut1, or PabPol Dmut2. DP1 was added at the same time as DP2 and at an equimolar ratio. Lane 9, $0.4 \mathrm{pmol}$ of $\mathrm{PabPolD}$. Reactions were incubated at $60^{\circ} \mathrm{C}$ for $30 \mathrm{~min}$, and products were resolved on denaturing alkaline 1\% agarose gels and visualized on a Typhoon 9400 imager.

Figure 6. PabPol Dmut1 and PabPol Dmut2 are less thermally stable than wild-type PabPol D. Nile Red fluorescence of PabPol D (a), PabPol Dmut1 (b), and PabPol Dmut2 (c). Enzymes were heated for $5 \mathrm{~min}$ and $48 \mathrm{pmol}$ incubated with Nile Red. The excitation wavelength was $550 \mathrm{~nm}$, and emission spectra were recorded in the range of 580-440 nm. The dotted line represents Nile Red alone. The double dot-dashed line represents unheated enzyme. The dot-dash line, dashed, and continuous lines represent enzyme heated at $80^{\circ} \mathrm{C}, 90^{\circ} \mathrm{C}$, and $100^{\circ} \mathrm{C}$, respectively. Intensity represents counts $\mathrm{x}$ $10^{6}$.

(d) PabPol D, PabPol Dmut1, and PabPol Dmut2 polymerase activity after heating. The enzyme was heated for $5 \mathrm{~min}$ at $80^{\circ} \mathrm{C}, 90^{\circ} \mathrm{C}$, or $100^{\circ} \mathrm{C}$ and 2 pmol incubated with $\mathrm{M} 13 \mathrm{mp} 18$ template (90 fmol) hybridized to a 5'-fluorescein-labeled primer for primer extension studies. Reactions were performed at $60^{\circ} \mathrm{C}$ for $30 \mathrm{~min}$, and products were resolved on denaturing alkaline $1 \%$ agarose gels and visualized on a Typhoon 9400 imager. 
Fig. 1

a)

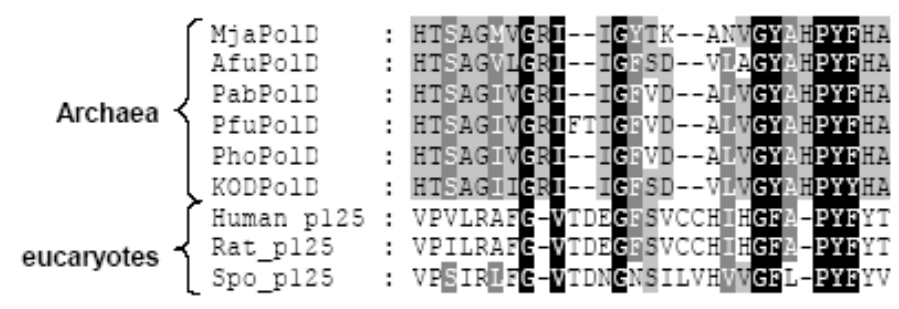

b)

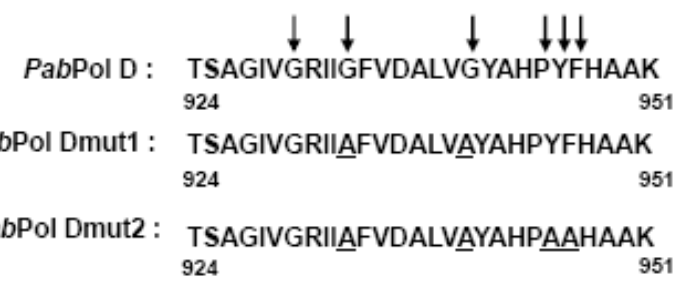


Fig. 2

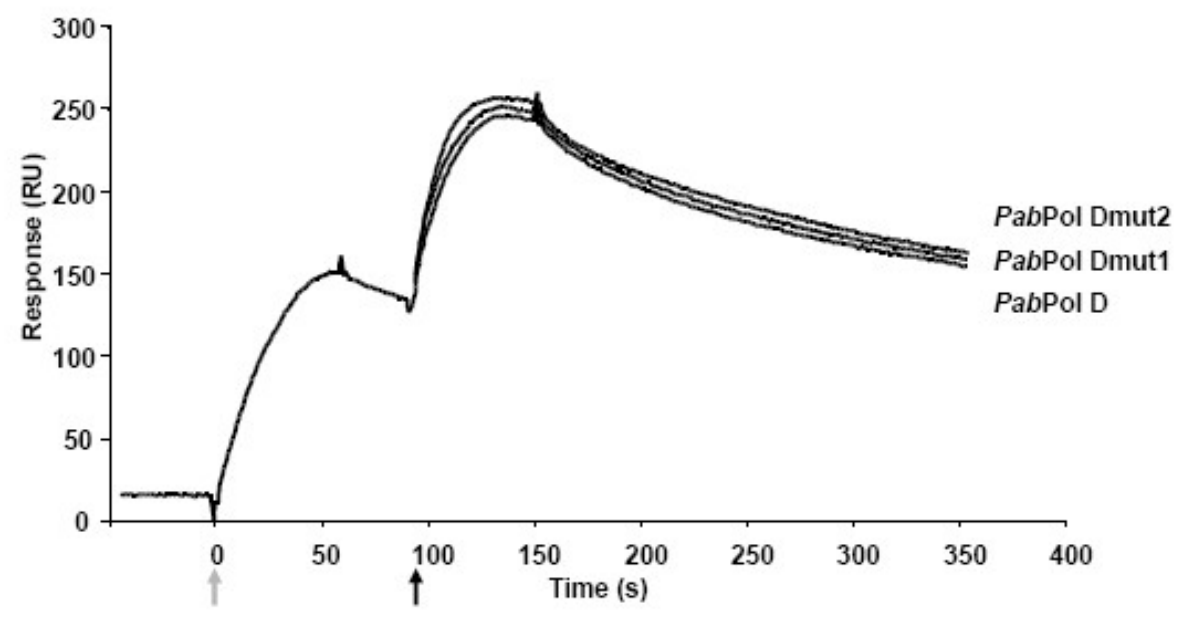


Fig. 3

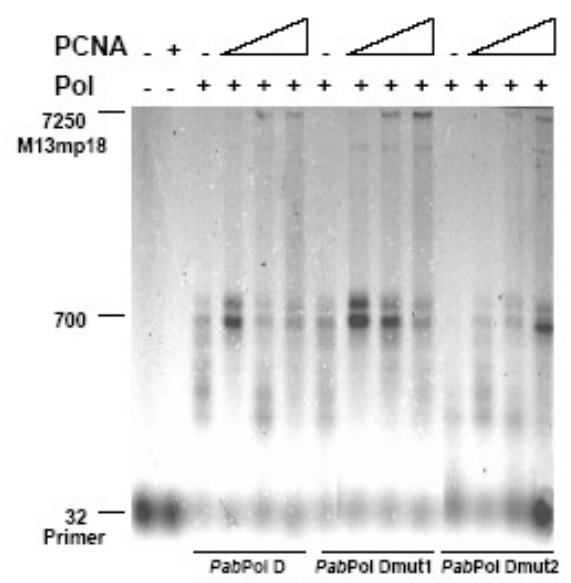


Figure4

Fig. 4

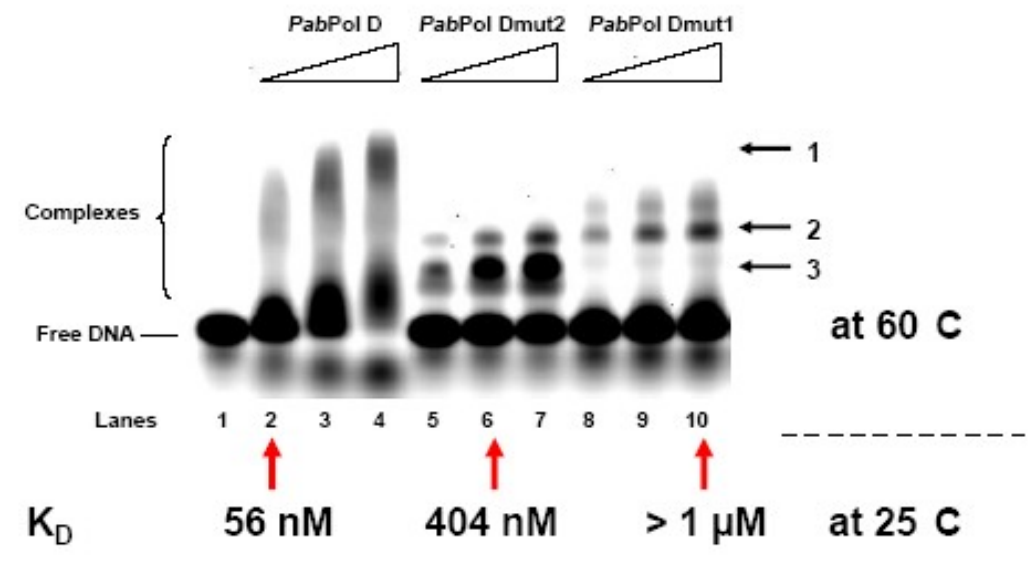


Figure5

Fig. 6

a)
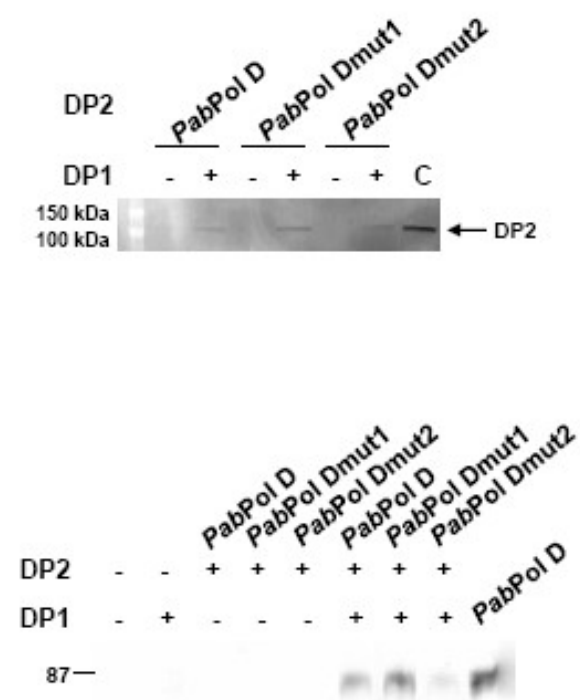

b)

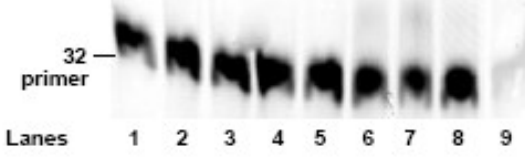


Fiprg. 6

a)

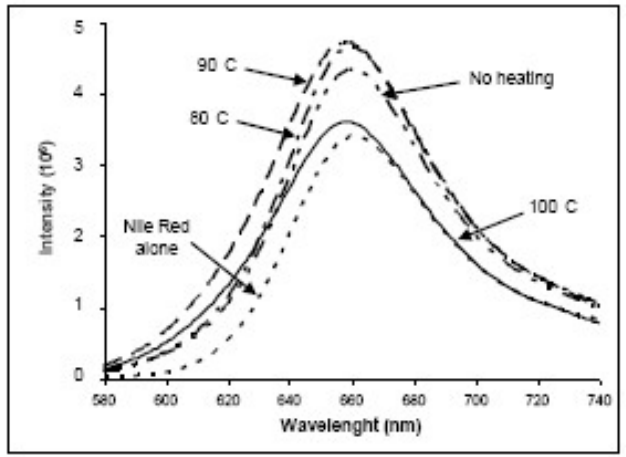

c)

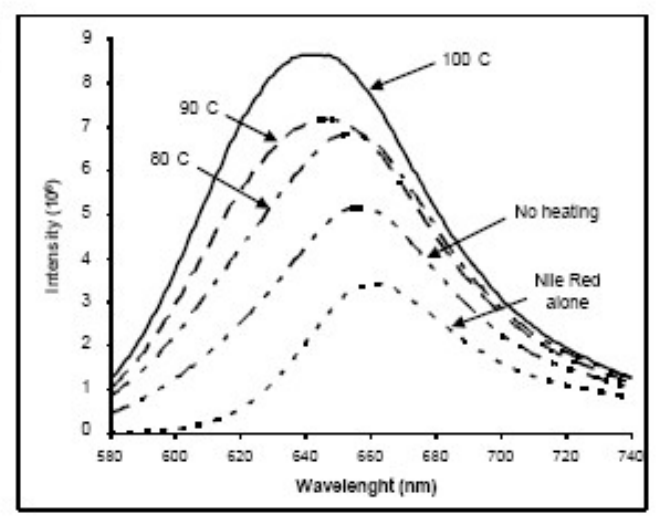

b)

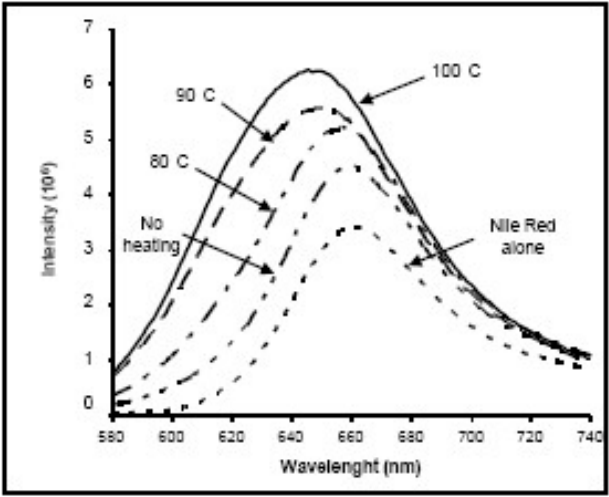

d)

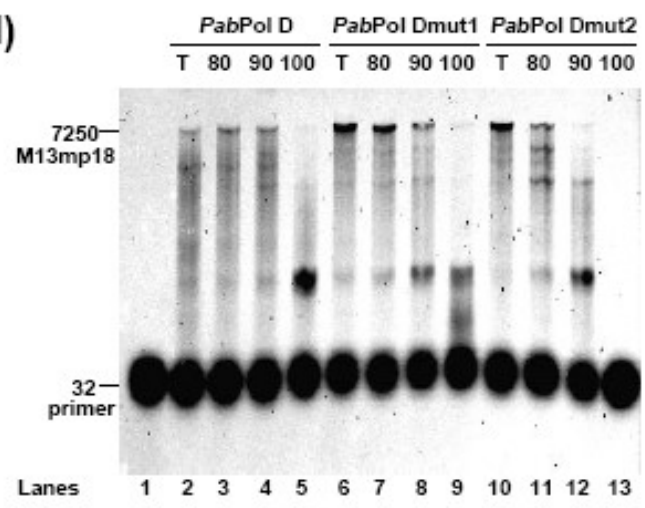

